\title{
Co-culture supernatants from Vibrio vulnificus-infected INT-407 cells induce IL-8 production in intestinal epithelial cells: Crucial role of $V$. vulnificus rtxE
}

\author{
BYUNG CHEOL LEE ${ }^{1}$, MYUN SOO KIM ${ }^{1}$, DAEHO CHO ${ }^{2}$, SANG HO CHOI ${ }^{3}$ and TAE SUNG KIM ${ }^{1}$ \\ ${ }^{1}$ Division of Life Sciences, School of Life Sciences and Biotechnology, Korea University; ${ }^{2}$ Department of Life Sciences, \\ Sookmyung Women's University; ${ }^{3}$ National Research Laboratory of Molecular Microbiology and Toxicology, \\ Department of Agricultural Biotechnology, Seoul National University, Seoul, Republic of Korea
}

Received June 8,2010; Accepted August 11, 2010

DOI: 10.3892/ijmm_00000510

\begin{abstract}
In a previous study, we reported that a gene mutation of repeat in toxin $\mathrm{E}$ (RtxE), a transporter of cytotoxic factors, resulted in a significant impairment of epithelial cell cytotoxicity in Vibrio vulnificus, and that the expression of the $r t x E$ gene was induced by the exposure to the host cells. In this study, we evaluated and compared the effects of coculture supernatants from V. vulnificus-infected INT-407 cells and either the $V$. vulnificus wild-type or $r t x E$ mutant on the production of interleukin (IL)-8, a pro-inflammatory cytokine, as well as its underlying mechanisms in human intestinal epithelial cells. INT-407 cells were co-cultured with the wild-type $V$. vulnificus or the $r t x E$ mutant strain to obtain the conditioned supernatants. IL-8 production and nuclear factor (NF)-кB activation from the INT-407 cells treated with each supernatant, were investigated. The co-culture supernatants from the rtxE mutant $V$. vulnificus-infected INT-407 cells significantly induced lower levels of IL- 8 production and promoter activation, $\mathrm{NF}-\kappa \mathrm{B}$ DNA binding activity, and NF- $\mathrm{BB}$ minimal promoter activation in human intestinal epithelial cells, than those from the wild-type $V$. vulnificus-infected INT-407 cells. Importantly, the reduced IL-8 production and $\mathrm{NF}-\kappa \mathrm{B}$ activity of the $V$. vulnificus $r t x E$ mutant, were restored by co-culture supernatants from the rtxE-complemented $V$. vulnificus. On the whole, these results show that the rtxE gene of $V$. vulnificus performs a critical role in the secretion of factors from bacteria and host cells, which are involved in IL-8 production via the NF- $\mathrm{KB}$ activation pathway in host cells.
\end{abstract}

Correspondence to: Dr Tae Sung Kim, Division of Life Sciences, School of Life Sciences and Biotechnology, Korea University, Seoul 136-701, Republic of Korea

E-mail: tskim@korea.ac.kr

Key words: Vibrio vulnificus, repeat in toxin, interleukin-8, nuclear factor- $\mathrm{\kappa} \mathrm{B}$, intestinal epithelial cell

\section{Introduction}

Vibrio vulnificus is a gram-negative estuarine bacterium, and has been identified as a significant human pathogen. Mortality rates from primary septicemia are quite high $(>50 \%)$ and death can occur within 1-2 days after the first signs of illness. $V$. vulnificus infection is characterized by septicemia and skin infections, accompanied in many cases by ulcers and edema $(1,2)$. The majority of fatal cases are caused by septic shock (3), which results from a variety of $V$. vulnificus virulence factors, including capsular polysaccharides (CPS) (4-6), hemolysin (7), siderophores (8), and repeats in toxin (RTX) (9-12).

The $V$. vulnificus RTX toxins are comprised of 4 genes of 2 rtx operons: $r t x A$ encodes for the toxin, $r t x C$ encodes for an essential acylase of RtxA, $r t x B / r t x E$ encodes for an ATPbinding cassette transporter of RtxA, and $r t x D$ encodes for a gene of unknown function (9). RtxE is a component of the type I secretion system (TISS), which is involved in the secretion of several virulence factors, such as RtxA, from the cells to the cell exterior (13-15). Previously, we determined that a gene mutation of the $r t x E$ gene in $V$. vulnificus can block the secretion of virulence factors, thereby resulting in a significant reduction in the cytotoxic activity of $V$. vulnificus. The expression of the $r t x E$ gene was shown to be induced by the exposure to the host cells $(10,11)$, thereby indicating that virulence factors can be secreted into the exterior via the RtxE-harboring TISS only after the bacteria make contact with the host cells.

Bacterial virulence factors, or the overproduction and dysregulation of the host cytokines during bacterial infection, result in septic shock or septicemia. These factors can persistently activate the generation of pro-inflammatory mediators including tumor necrosis factor- $\alpha$, interleukin (IL)-1ß, IL-6, IL-8 and nitric oxide in the infected host $(16,17)$.

The majority of these mediators show evidence of multiple biological effects, and have been identified as key mediators in the pathophysiological alterations noted in septic shock (18-20). Immunotherapeutic intervention strategies that abrogate the biological activities of these mediators have been observed to exert a significant protective effect against the lethal effects of LPS $(21,22)$. Previously, we reported that 
V. vulnificus infection induced IL-8 production in human intestinal epithelial cells via the nuclear factor (NF)- $\mathrm{B}$ activation pathway (17). IL-8 has also been shown to play a critical role in the pathogenesis of infectious diseases.

Therefore, in this study, in order to evaluate the role of RtxE in IL-8 production in human epithelial cells, we investigated and compared the effects of co-culture supernatants from host INT-407 cells and either the wild-type or the $r t x E$ mutant of $V$. vulnificus on IL-8 production, as well as their underlying mechanisms. We demonstrated that RtxE, a cytotoxic factor transporter, performs a critical function in the secretion of factors, which are involved in IL-8 production via the NF- $\mathrm{KB}$ activation pathway in human intestinal epithelial cells.

\section{Materials and methods}

Cell cultures. Human intestinal epithelial cell-lines, INT-407 cells, were purchased from the American Type Culture Collection (Manassas, VA, USA) and maintained at $37^{\circ} \mathrm{C}$ in $5 \% \mathrm{CO}_{2}$ in Minimum Essential Medium (MEM) supplemented with $10 \%$ fetal bovine serum (Gibco BRL, Grand Island, NY, USA) and antibiotics (10 units/ml penicillin $\mathrm{G}$ and $10 \mu \mathrm{g} / \mathrm{ml}$ streptomycin) (growth medium).

Bacterial strain and growth conditions. The $V$. vulnificus strain, MO6-24/O, used in this study was isolated from clinical patients, and the $r t x E$ gene mutant and the $r t x E$-complemented strains were generated, as previously described (11). For the infection experiments, the bacteria were grown at $30^{\circ} \mathrm{C}$ in Luria-Bertani medium supplemented with $2 \% \mathrm{NaCl}$ (LBS medium), and diluted to $\sim 6 \times 10^{8} \mathrm{CFU} / \mathrm{ml}$ in LBS, then centrifuged and resuspended in antibiotic-free growth medium prior to the epithelial cell infection. Bacterial concentrations were confirmed via viable cell counts on LBS agar.

Infection protocol. Human intestinal epithelial cells were infected with $V$. vulnificus, as previously described $(11,12)$. In brief, intestinal epithelial cells were grown in culture flasks at $37^{\circ} \mathrm{C}$ in a $5 \% \mathrm{CO}_{2}$ incubator. The cells $\left(3 \times 10^{6}\right)$ were seeded onto culture dishes $(10 \mathrm{ml}$ medium $/ 10-\mathrm{cm})$ and incubated for $24 \mathrm{~h}$ in antibiotic-free growth medium. Prior to infection, the bacteria were centrifuged for $3 \mathrm{~min}$ at 2,500 x g, resuspended and adjusted to $\sim 6 \times 10^{8} \mathrm{CFU} / \mathrm{ml}$ in antibiotic and phenol redfree MEM. The bacterial suspensions were added to the epithelial cells at a multiplicity of infection (ratio of no. of bacteria to no. of epithelial cells) of 10 , after which the infected cells were incubated in a $5 \% \mathrm{CO}_{2}$ incubator for $2 \mathrm{~h}$ at $37^{\circ} \mathrm{C}$ in antibiotic and phenol red-free growth medium.

Preparation of co-culture supernatants from V. vulnificusinfected INT-407 cells. Co-culture supernatants were obtained and harvested by centrifugation at 4,000 x g for $10 \mathrm{~min}$ at $4^{\circ} \mathrm{C}$. This was repeated once more with the resulting supernatant. The clarified supernatants were filtered through a $0.22 \mu \mathrm{M}$ pore membrane to ensure the removal of any remaining bacterial cells and debris.

Transient transfection and dual luciferase reporter assay. Human intestinal epithelial cells were transiently transfected with human IL-8 full promoter and IL-8/linker scanning (LS) mutation promoter, with $\mathrm{NF}-\kappa \mathrm{B}$ minimal promoter constructs, or pRL-TK reporter vector, as previously described (6). In brief, intestinal epithelial cells were plated at a density of $2 \times 10^{4}$ cells in 24 -well plates. After $24 \mathrm{~h}$ of culture, the INT407 cells were transiently transfected with each of the human IL-8 full, IL-8/LS promoter constructs, NF- $\kappa \mathrm{B}$ minimal promoter, or the pRL-TK Renilla luciferase reporter vector (Promega) by using the SuperFect method (Qiagen, Valencia, CA, USA), according to the manufacturer's instructions. The transfected cells were incubated for $12 \mathrm{~h}$ at $37^{\circ} \mathrm{C}$ in a $5 \% \mathrm{CO}_{2}$ incubator. The cells were washed in PBS, after which the cells were incubated with culture supernatants for 12 or $24 \mathrm{~h}$. Afterwards, the cells were harvested and suspended for $20 \mathrm{~min}$ in $100 \mu \mathrm{l}$ of lysis buffer. The supernatant fluid was harvested and assayed for luciferase activity using Dual Luciferase Reagent (Promega) in a luminometer. Results were normalized by Renilla luciferase activity, and were expressed as relative fold induction.

Reverse transcription polymerase chain reaction ( $R T-P C R)$. Total RNA was prepared from the cells and reverse-transcribed into cDNA, after which the cDNA was amplified by PCR. Total cellular RNA was isolated via the single-step method, using easy-Blue reagent (Intron, Korea). The primers used were as follows: Human IL-8 (302 bp), 5'-ATGACTT CCAAGCTGGCCGTGGCT-3' (sense) and 5'-TTATGAATT CTCAGCCCTCTTCAAAAACTTCTC-3' (antisense); and Bactin (373 bp), 5'-TTCTACAATGAGCTGCGTGTGGCT-3' (sense) and 5'-GCTTCTCCTTAATGTCACGCACGA-3' (antisense). The reactions were conducted in an MJ Thermal Cycler (Watertown, MA, USA) for 34 cycles of the following: Denaturing at $94^{\circ} \mathrm{C}$ for $30 \mathrm{sec}$, annealing at $60^{\circ} \mathrm{C}$ for $30 \mathrm{sec}$, and extending at $72^{\circ} \mathrm{C}$ for $30 \mathrm{sec}$. After amplification, the RT-PCR products were separated on $2 \%(\mathrm{w} / \mathrm{v})$ agarose gels and stained with ethidium bromide.

Preparation of cell lysates and Western blot analysis. The cells were lysed in lysis buffer (50 mM Tris buffer, $\mathrm{pH} 7.5$, containing $100 \mathrm{mM} \mathrm{NaCl}, 1 \%$ Nonidet P-40, 10\% glycerol, $1 \mathrm{mM}$ EDTA, $1 \mathrm{mM} \mathrm{NaF}, 1 \mathrm{mM}$ sodium orthovanadate, $50 \mu \mathrm{g} / \mathrm{ml}$ each of leupeptin, aprotinin and PMSF) by incubation on ice for $30 \mathrm{~min}$. The lysates were then centrifuged at $13,000 \mathrm{x}$ g at $4^{\circ} \mathrm{C}$ for $15 \mathrm{~min}$, and the supernatants were transferred to fresh tubes and stored at $-70^{\circ} \mathrm{C}$ until use. Protein concentrations of the lysates were determined by using the $\mathrm{BCA}^{\mathrm{TM}}$ Protein Assay Reagent A (Pierce, USA). Equal amounts $(20 \mu \mathrm{g} / \mathrm{ml})$ of whole cell lysates were subjected to sodium dodecyl sulfate-10\% polyacrylamide gel electrophoresis (SDS-PAGE). The proteins were transferred onto a polyvinylidene fluoride membrane using the Semi-Phor unit (Hoefer Scientific Instruments). The membrane was then incubated with washing buffer (PBS solution containing 0.1\% Tween-20) containing $1 \%$ bovine serum albumin for at least $1 \mathrm{~h}$ to block non-specific protein binding. Afterwards, the membrane was, respectively, treated with anti- $\beta$-actin, anti-IкB $\alpha$ and antiphospho-IкB $\alpha$ antibodies (Santa Cruz Biotechnology, Inc.). After incubation with HRP-conjugated anti-rabbit antibody, immunoreactive proteins were detected with the ECL system (Amersham Biosciences, England). 
IL-8 ELISA. Human intestinal epithelial cells were cultured for $24 \mathrm{~h}$ in $60-\mathrm{mm}$ culture dishes prior to the infection with $V$. vulnificus. The intestinal epithelial cells were treated with co-culture supernatants from V. vulnificus-infected INT-407 cells for 12 or $24 \mathrm{~h}$. The culture supernatants were then analyzed for the levels of IL-8 protein using the OptEIA ${ }^{\text {TM }}$ human IL-8 ELISA kit 2 (BD Pharmingen, San Diego, CA, USA), according to the manufacturer's instructions. The absorbance at $490 \mathrm{~nm}$ of each well in 96-well plates, was determined using an ELISA reader.

Preparation of nuclear extracts and electrophoretic mobility shift assay (EMSA). Human intestinal epithelial cells $\left(8 \times 10^{5}\right.$ cells/dish) were seeded onto 60-mm culture dishes, cultured for $24 \mathrm{~h}$ in antibiotic-free growth medium, and then treated with co-culture supernatants for $1 \mathrm{~h}$. The nuclear extracts were prepared as previously described (6). In brief, the infected cells were washed twice in PBS and resuspended in hypotonic buffer (10 mM HEPES buffer, $\mathrm{pH} 7.9$, containing $0.5 \mathrm{mM}$ $\mathrm{KCl}, 1.5 \mathrm{mM} \mathrm{MgCl}_{2}, 0.5 \mathrm{mM}$ DTT and $0.2 \mathrm{mM}$ PMSF). After the cells were allowed to swell on ice for $10 \mathrm{~min}$, a $10 \%$ solution of Nonidet P-40 was added and the cells were maintained for $15 \mathrm{~min}$ on ice. The homogenates were centrifuged for $15 \mathrm{~min}$ at 5,000 $\mathrm{x} \mathrm{g}$, and the resultant nuclear pellets were resuspended in $50 \mu \mathrm{l}$ of low salt buffer (20 mM HEPES buffer, $\mathrm{pH} 7.9$, containing $25 \%$ glycerol, $1.5 \mathrm{mM} \mathrm{MgCl}_{2}$ and $0.2 \mathrm{mM}$ PMSF) and then $50 \mu \mathrm{l}$ of high salt buffer $(20 \mathrm{mM}$ HEPES buffer, pH 7.9, containing 25\% glycerol, $1.5 \mathrm{mM}$ $\mathrm{MgCl}_{2}, 0.8 \mathrm{M} \mathrm{KCl}, 0.2 \mathrm{mM}$ EDTA, $0.5 \mathrm{mM}$ DTT and $0.2 \mathrm{mM}$ PMSF) in a dropwise fashion. The nuclear extracts were centrifuged for $30 \mathrm{~min}$ at $20,500 \mathrm{x} \mathrm{g}$ at $4^{\circ} \mathrm{C}$. The nuclear extracts $(10 \mu \mathrm{g}$ of each one) were incubated for $30 \mathrm{~min}$ with the labeled oligonucleotides in $20 \mu$ l of binding buffer $(10 \mathrm{mM}$ Tris-HCl, pH 7.6, 500 mM KCl, 10 mM EDTA, 50\% glycerol, $250 \mathrm{ng}$ poly $(\mathrm{dI}-\mathrm{dC})$ and $1 \mathrm{mM}$ dithiothreitol). The reaction mixture was analyzed by electrophoresis on a $4 \%$ polyacrylamide gel in $0.5 \mathrm{X}$ Tris borate buffer. An oligonucleotide harboring an NF- $\kappa \mathrm{B}$ binding site (5'-CCGGTTAACAGA GGGGGCTTTCCGAG-3') was used as the probe. Specific binding was verified via competition experiments with a 50fold excess of unlabeled, identical oligonucleotides of cAMP response element (CRE)-containing oligonucleotides.

Fluorescence microscopy. For fluorescence assay, human intestinal epithelial cells were seeded onto 24-well plates and treated for $3 \mathrm{~h}$ with different co-culture supernatants from V. vulnificus-infected INT-407 cells. The cells were washed with PBS, fixed with $4 \%$ paraformaldehyde for $30 \mathrm{~min}$ at room temperature, permeabilized for $10 \mathrm{~min}$ with $0.2 \%$ Triton $\mathrm{X}-100$ in PBS, and blocked for $1 \mathrm{~h}$ with $1.5 \%$ fetal bovine serum in PBS. The cells were incubated with a rabbit anti$\mathrm{NF}-\kappa \mathrm{B}$ p65 antibody (diluted 1:200) for $1 \mathrm{~h}$ at room temperature, and then incubated with fluorescein isothiocyanateconjugated secondary antibody (Santa Cruz Biotechnology). The cells were mounted with anti-fade mounting medium and examined using an Olympus IX71 fluorescence microscope.

Statistical analyses. Student's $t$-tests and one-way analysis of variance (ANOVA), followed by the Bonferroni method, were employed in order to determine statistical differences between the values of the various experimental and control groups. $\mathrm{P}$-values of $<0.05$ were considered statistically significant.

\section{Results}

Co-culture supernatants from INT-407 cells infected by the $V$. vulnificus rtxE mutant significantly reduce IL-8 production in human intestinal epithelial cells. IL-8 is a pro-inflammatory cytokine involved in the pathogenesis of $V$. vulnificus. Factors affecting IL-8 production can be secreted into the supernatant via the RtxE-harboring TISS when $V$. vulnificus makes direct contact with the host cells.

In this study, we wanted to evaluate whether co-culture supernatants from $V$. vulnificus-infected INT-407 cells can induce IL-8 production in intestinal epithelial cells. As we have described previously, the $V$. vulnificus $r t x E$ gene plays a very crucial role in releasing factors into supernatants for IL-8 production in intestinal epithelial cells. Afterward, the supernatants were obtained, filtered, and utilized in further experiments. Co-culture supernatants from INT-407 cells infected with the $V$. vulnificus wild-type were used as the control. INT-407 cells were then treated with each of the coculture supernatants, and the expression levels of IL-8 mRNA and protein were determined.

As shown in Fig. 1, co-culture supernatants from the $r t x E$ mutant $V$. vulnificus-infected INT-407 cells significantly reduced IL-8 mRNA in intestinal epithelial cells. In contrast, the expression of $B$-actin mRNA was unaffected in the INT407 cells treated with the co-culture supernatants of the $\operatorname{rtxE}$ gene mutant and host cells, thereby indicating that the reduced effects of the $r t x E$ gene mutation on IL-8 expression were not the consequence of a general dampening of cellular activation (Fig. 1A). Additionally, co-culture supernatants from rtxE mutant $V$. vulnificus-infected INT-407 cells significantly reduced IL-8 production in intestinal epithelial cells (Fig. 1B). The growth rates of $2 \mathrm{~V}$. vulnificus strains (the wild-type and $r t x E$ mutant of $V$. vulnificus) did not differ significantly (data not shown).

Co-culture supernatants from rtxE mutant V. vulnificusinfected INT-407 cells significantly reduce IL-8 promoter activation in human intestinal epithelial cells. In order to determine whether co-culture supernatants from either the $r t x E$ mutant or the wild-type $V$. vulnificus-infected INT-407 cells differentially affect the activity of the IL-8 gene promoter, human intestinal epithelial cells were transiently transfected with the IL-8 promoter/luciferase construct (IL-8 full promoter, Fig. 2A), and then treated with co-culture supernatants from the $r t x E$ mutant or wild-type, after which the luciferase activity was assessed. As shown in Fig. 2B, the IL-8 full promoter construct was significantly stimulated with co-culture supernatants from the wild-type $V$. vulnificusinfected INT-407 cells. However, the stimulated levels were significantly lower in the co-culture supernatants from the rtxE mutant-infected INT-407 cells compared to those treated with co-culture supernatants from the wild-type $V$. vulnificusinfected INT-407 cells, thus indicating that co-culture supernatants from the rtxE mutant $V$. vulnificus-infected INT-407 cells lacked the factor(s) involved in the activation of the IL-8 promoter. 
$\mathbf{A}$

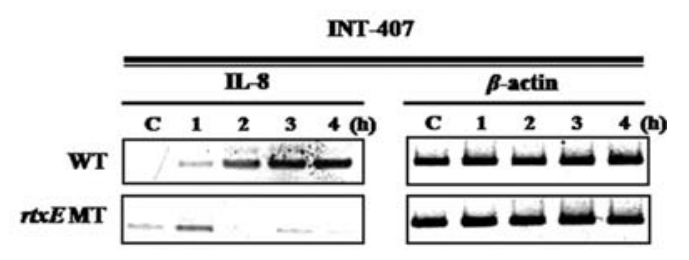

B

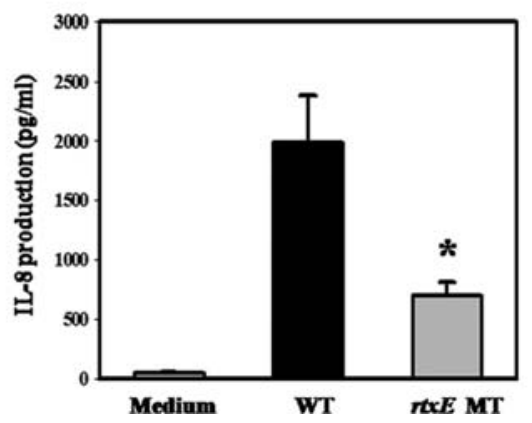

Figure 1. Effects of co-culture supernatants from rtxE mutant $V$. vulnificusinfected INT-407 cells on IL-8 production. (A) Human intestinal epithelial cells were treated for 1-4 h with co-culture supernatants from the WT or rtxE MT V. vulnificus-infected INT-407 cells. Cellular RNA was extracted at each time-point, and IL-8 mRNA expression was determined by RT-PCR. (B) Human intestinal epithelial cells were treated for $24 \mathrm{~h}$ with co-culture supernatants (1.5 ml/well) from WT or rtxE MT V. vulnificus-infected INT407 cells. The levels of IL- 8 protein were analyzed by ELISA. The data are representative of 3 independent experiments. The data are expressed as the means \pm standard errors $(n=3)$. ${ }^{*} \mathrm{P}<0.01$, relative to the group treated with co-culture supernatants from the WT $V$. vulnificus-infected INT-407 cells. WT, wild-type; $r t x E$ MT, $r t x E$ mutant.

NF-кB, NF-IL-6 and AP-1 are 3 transcriptional factors that are crucial for the regulation of IL- 8 gene expression $(23,24)$. Previously, we determined that the NF-кB site was required for IL-8 production after $V$. vulnificus infection in human intestinal epithelial cells $(6,17)$. In order to directly investigate the role of a NF-кB site located between -80 and -71 of the IL- 8 full promoter in co-culture supernatantstimulatory action, we introduced an LS mutation into the NF- $\kappa$ B site within the context of the $-144 /+44$ construct (IL-8/ LS promoter, Fig. 2A). As shown in Fig. 2B, the activation of co-culture supernatants from the wild-type $V$. vulnificusinfected INT-407 cell-dependent promoter, was significantly reduced, although it was still observed with the IL-8/LS promoter. The IL-8/LS promoter was not activated when the cells were treated with co-culture supernatants from the rtxE mutant $V$. vulnificus-infected INT-407 cells. These results indicate that the $\operatorname{rtx} E$ gene of $V$. vulnificus plays a role in secreting factors from INT-407 cells and that $V$. vulnificus is involved in IL-8 production via the NF- $\mathrm{KB}$ activation pathway when the bacteria are co-cultured with host cells.

Co-culture supernatants from rtxE mutant $V$. vulnificusinfected INT-407 cells significantly reduce $N F-\kappa B D N A$ binding activity and $I \kappa B \alpha$ phosphorylation compared to those from wild-type V. vulnificus-infected INT-407 cells. To determine whether NF- $\mathrm{\kappa B}$ activity was relevant to the reduced levels of IL-8 production in human epithelial cells treated with co-culture supernatants from rtxE mutant $V$. vulnificus- infected INT-407 cells, human intestinal epithelial cells were transiently transfected with $\mathrm{NF}-\kappa \mathrm{B}$ minimal promoter/ luciferase construct, followed by treatment with co-culture supernatants from V. vulnificus-infected INT-407 cells, and then the luciferase activity was determined. The NF-кB minimal promoter construct was potently stimulated with coculture supernatants from the wild-type $V$. vulnificus-infected INT-407 cells in a time-dependent fashion. However, the levels of NF- $\mathrm{KB}$ activation were significantly reduced in the co-culture supernatants from the rtxE mutant $V$. vulnificusinfected INT-407 cells (Fig. 3A).

Also, in order to ascertain whether or not NF- $\kappa \mathrm{B}$ activity was involved in the reduced levels of IL- 8 production in intestinal epithelial cells treated with co-culture supernatants from rtxE mutant $V$. vulnificus-infected INT-407 cells, we analyzed the NF-кB DNA binding activity in the nuclear extracts of intestinal epithelial cells treated with each of the co-culture supernatants. As shown in Fig. 3B, the treatment of intestinal epithelial cells with co-culture supernatants from the wild-type $V$. vulnificus-infected INT-407 cells resulted in profound $\mathrm{NF}-\kappa \mathrm{B}$ binding activity. As we anticipated, the treatment with co-culture supernatants from the $r t x E$ mutant V. vulnificus-infected INT-407 cells significantly reduced NF- $\mathrm{B}$ DNA binding activity in human intestinal epithelial cells. This result was in contrast to the one observed with the co-culture supernatants from the wild-type V. vulnificusinfected INT-407 cells, as determined by electrophoretic mobility shift assays using labeled oligonucleotides containing a consensus NF- $\mathrm{KB}$ site. This binding was recognized as specific, as it competed with an unlabeled, identical oligonucleotide, but not with an unrelated, non-specific oligonucleotide, and was absent in the nuclear extracts from the non-stimulated cells.

In the majority of cells, $\mathrm{NF}-\kappa \mathrm{B}$ exists in an inactive form in the cytoplasm, bound to the inhibitory I $\mathrm{B} \alpha \alpha$ proteins. The treatment of cells with various inducers results in the degradation of the IкB $\alpha$ proteins. The bound $\mathrm{NF}-\kappa \mathrm{B}$ is then released and translocated into the nucleus, where it activates the appropriate target genes. In order to further assess the involvement of $\mathrm{I} \kappa \mathrm{B} \alpha$ degradation in the reduced $\mathrm{NF}-\kappa \mathrm{B}$ binding activity observed with co-culture supernatants from rtxE mutant $V$. vulnificus-infected INT-407 cells, we determined the levels of the $\mathrm{I} \kappa \mathrm{B} \alpha$ protein via Western blot analysis using anti-IкB $\alpha$ $\mathrm{mAb}$ and anti-phospho-IкB $\alpha$ antibodies. As shown in Fig. 3C, co-culture supernatants from the rtxE mutant $V$. vulnificusinfected INT-407 cells significantly induced lower levels of IкB $\alpha$ protein in human intestinal epithelial cells compared to those from the wild-type V. vulnificus-infected INT-407 cells. Additionally, co-culture supernatants from the $r t x E$ mutant $V$. vulnificus-infected INT-407 induced lower levels of $\mathrm{I} \kappa \mathrm{B} \alpha$ phosphorylation compared to those from the wildtype V. vulnificus-infected INT-407 cells (Fig. 3C). These data clearly indicate that co-culture supernatants from wildtype V. vulnificus-infected INT-407 cells induce IL-8 production in human intestinal epithelial cells via the NF-кB pathway, and that the reduced IL- 8 production in human intestinal epithelial cells treated with co-culture supernatants from rtxE mutant $V$. vulnificus-infected INT-407 cells, resulted from the down-regulation of the NF-кB activation pathway. 
$\mathbf{A}$

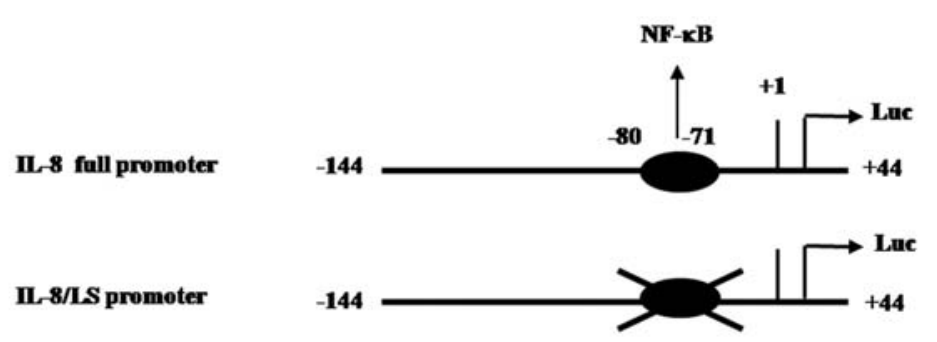

B
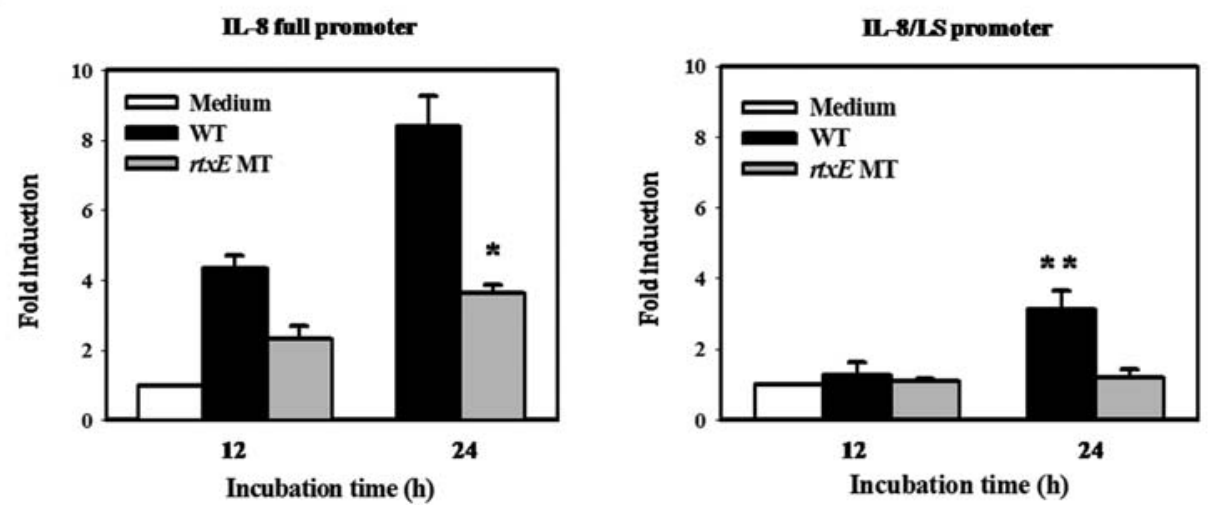

Figure 2. Effects of co-culture supernatants from rtxE MT V. vulnificus-infected INT-407 cells on IL-8 promoter activity. (A) Schematic representation of the IL-8 promoter constructs. (B) Human intestinal epithelial cells were transiently co-transfected with the IL-8 full promoter/luciferase construct (IL-8 full promoter) or the IL-8/LS promoter construct and pRL-TK luciferase reporter vector, followed by 12 or $24 \mathrm{~h}$ of treatment with co-culture supernatants from WT or $r t x E$ MT V. vulnificus-infected INT-407 cells. Afterwards, the luciferase activity was determined by a dual luciferase assay. The results were normalized by Renilla luciferase activity, and are expressed as the relative fold induction. The data are expressed as the means \pm standard errors ( $\mathrm{n}=3$ ). The data are representative of 3 independent experiments. ${ }^{*} \mathrm{P}<0.01$, relative to the group treated with co-culture supernatants from WT $V$. vulnificus-infected INT-407 cells for 24 h. ${ }^{* *} \mathrm{P}<0.05$, relative to an uninfected group treated with medium alone. WT, wild-type; rtxE MT, rtxE mutant.

A

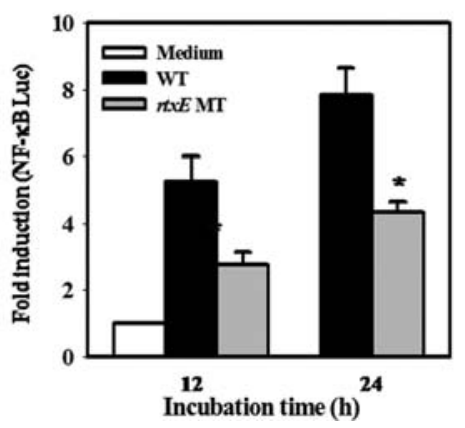

B

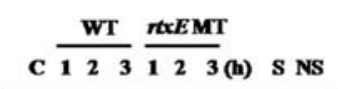

C

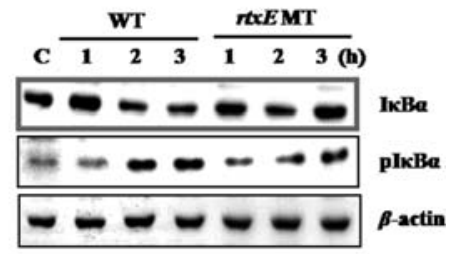

Figure 3. Effects of co-culture supernatants from $r t x E$ MT $V$. vulnificus-infected INT-407 cells on NF- $\mathrm{B}$ DNA activation and IкB $\alpha$ phosphorylation. (A) Human intestinal epithelial cells were transiently co-transfected with the NF- $\mathrm{B}$ minimal promoter/luciferase construct and pRL-TK vector, followed by 12 or $24 \mathrm{~h}$ of treatment with co-culture supernatants from WT or rtxE MT V. vulnificus-infected INT-407 cells. Afterwards, the cells were washed with PBS and the luciferase activity was assessed. The data are representative of 3 independent experiments. The data are expressed as the means \pm standard errors ( $=3$ ). ${ }^{*} \mathrm{P}<0.01$, relative to the group treated with co-culture supernatants from WT $V$. vulnificus-infected INT-407 cells at each time-point. The results were normalized by Renilla luciferase activity, and are expressed as the relative fold induction. (B) Human intestinal epithelial cells were treated for 1-3 $\mathrm{h}$ with coculture supernatants from WT or rtxE MT V. vulnificus-infected INT-407 cells. The nuclear extracts were assessed for NF-кB DNA binding activity by EMSA. S and NS indicate the presence of a 50-fold excess of specific oligonucleotide (NF- $\mathrm{B}$ ) and non-specific oligonucleotide (CRE-containing oligonucleotide), respectively. (C) Human intestinal epithelial cells were treated for 1-3 h with co-culture supernatants from WT or rtxE MT $V$. vulnificus-

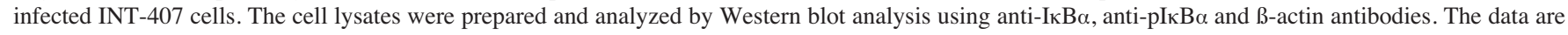
representative of 3 independent experiments. WT, wild-type; rtxE MT, rtxE mutant.

The reduced levels of $I L-8$ production and gene promoter activation were restored by co-culture supernatants from rtxE gene-complemented V. vulnificus-infected INT-407 cells. In order to determine whether co-culture supernatants from $r t x E$ gene-complemented $V$. vulnificus-infected INT-407 cells ameliorate the reduced IL- 8 production induced by co-culture 
$\mathbf{A}$

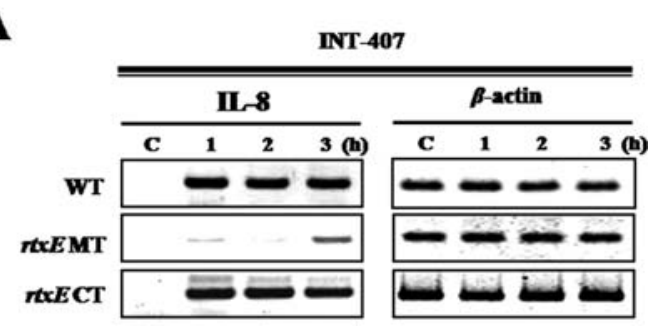

B

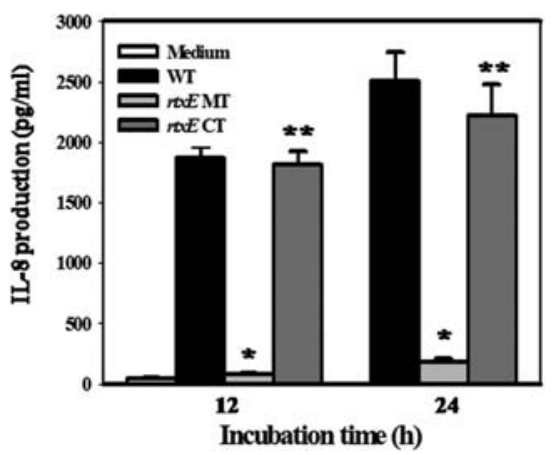

Figure 4. Effects of co-culture supernatants from rtxE CT V. vulnificusinfected INT-407 cells on IL-8 production. (A) Human intestinal epithelial cells were treated for $1-3 \mathrm{~h}$ with co-culture supernatants $(1.5 \mathrm{ml} / 6-\mathrm{cm}$ dish $)$ from WT, rtxE MT, or rtxE CT $V$. vulnificus-infected INT-407 cells. IL-8 mRNA expression was determined by RT-PCR. (B) Human intestinal epithelial cells were treated for 12 or $24 \mathrm{~h}$ with co-culture supernatants from WT, rtxE MT, or rtxE CT V. vulnificus-infected INT-407 cells. The culture supernatants were analyzed for IL-8 protein levels by ELISA. The data are representative of 3 independent experiments. The data are expressed as the means \pm standard errors $(n=3)$. ${ }^{*} \mathrm{P}<0.001$, relative to the group treated with co-culture supernatants from WT $V$. vulnificus-infected INT-407 cells at each time-point. ${ }^{* *} \mathrm{P}<0.01$, relative to the group treated with co-culture supernatants from $r t x E$ MT $V$. vulnificus-infected INT-407 cells at each time-point. WT, wild-type; $r t x E \mathrm{MT}$, rtxE mutant; $r t x E \mathrm{CT}$, rtxE-complemented.

supernatants from rtxE mutant $V$. vulnificus-infected INT407 cells, human intestinal epithelial cells were treated with co-culture supernatants from wild-type, $r t x E$ mutant, or $r t x E$ complemented $V$. vulnificus-infected INT-407 cells, after which the levels of IL- 8 expression were evaluated. As is shown in Fig. 4, co-culture supernatants from the $r t x E$ mutant V. vulnificus-infected INT-407 cells significantly induced lower levels of IL-8 mRNA and protein than those from the wild-type $V$. vulnificus-infected INT-407 cells in human intestinal epithelial cells. Importantly, the reduced levels of IL-8 production were restored by the co-culture supernatants from the rtxE gene-complemented $V$. vulnificus-infected INT-407 cells.

In order to further determine whether co-culture supernatants from rtxE-complemented $V$. vulnificus-infected INT407 cells could restore the IL- 8 promoter activity reduced by the co-culture supernatants from rtxE mutant $V$. vulnificusinfected INT-407 cells, human intestinal epithelial cells were transiently co-transfected with the IL-8 full promoter/ luciferase and pRL-TK luciferase reporter vectors, and were then treated with co-culture supernatants from wild-type, $r t x E$ mutant, or rtxE-complemented $V$. vulnificus-infected INT-407 cells. IL-8 promoter activity was then determined
$\mathbf{A}$

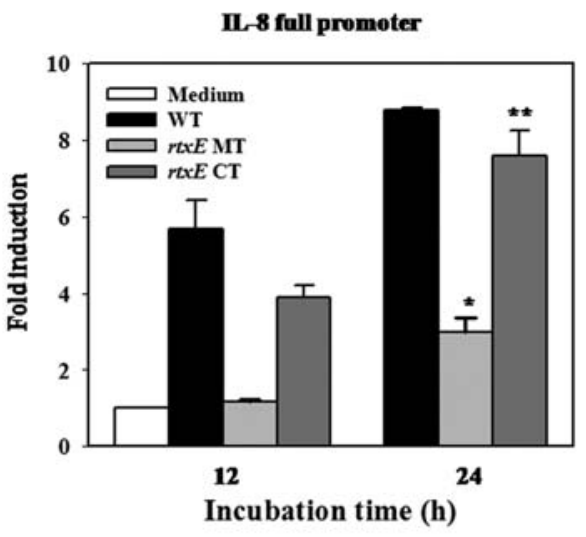

B

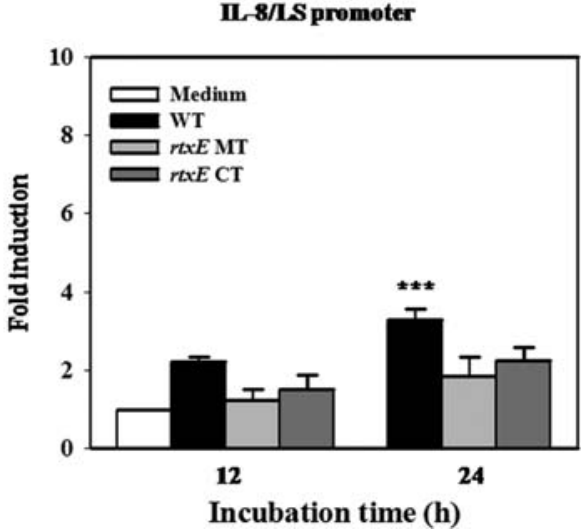

Figure 5. Effects of co-culture supernatants from rtxE CT V. vulnificusinfected INT-407 cells on IL-8 promoter activity. (A) Human intestinal epithelial cells were transiently co-transfected with the IL-8 full promoter or the IL-8/LS promoter construct and pRL-TK luciferase reporter vector, followed by treatment with co-culture supernatants from WT, rtxE MT, or rtxE CT V. vulnificus-infected INT-407 cells for 12 or $24 \mathrm{~h}$. Afterwards, the luciferase activity was determined. The results were normalized by Renilla luciferase activity, and are expressed as the relative fold induction. The data are representative of 3 independent experiments. The data are expressed as the means \pm standard errors $(n=3) .{ }^{*} \mathrm{P}<0.01$, relative to the group treated with co-culture supernatants from WT $V$. vulnificus-infected INT-407 cells at $24 \mathrm{~h}$. ${ }^{* *} \mathrm{P}<0.05$, relative to the group treated with co-culture supernatants from rtxE MT V. vulnificus-infected INT-407 cells at $24 \mathrm{~h}$. ${ }^{* * *} \mathrm{P}<0.01$, relative to an uninfected group incubated with medium alone. WT, wild-type; rtxE MT, $r t x E$ mutant; rtxE CT, rtxE-complemented.

by a dual luciferase assay. As shown in Fig. 5, co-culture supernatants from the rtxE mutant $V$. vulnificus-infected INT-407 cells significantly induced lower levels of IL-8 promoter activation compared to those from the wild-type $V$. vulnificusinfected INT-407 cells. In contrast, co-culture supernatants from the rtxE-complemented $V$. vulnificus-infected INT-407 cells induced similar levels of IL-8 promoter activation as those noted with co-culture supernatants from the wild-type V. vulnificus-infected INT-407 cells, thereby indicating that the reduced IL-8 promoter activity was restored by co-culture supernatants from the rtxE-complemented $V$. vulnificusinfected INT-407 cells (Fig. 5). The levels of IL-8/LS promoter activity were approximately the same in human intestinal epithelial cells treated with co-culture supernatants from the V. vulnificus wild-type, rtxE mutant, or $r t x E$ complemented strains. 
$\mathbf{A}$

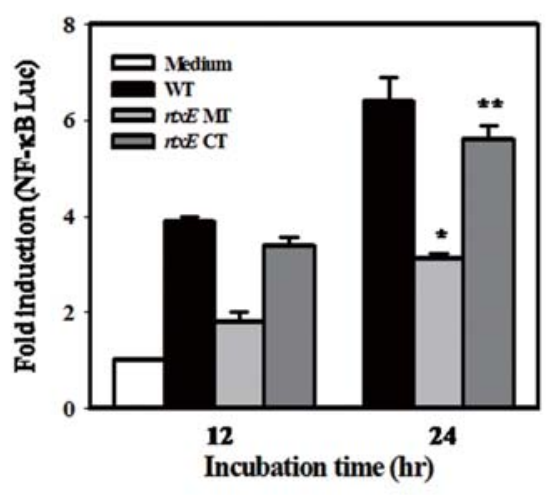

C

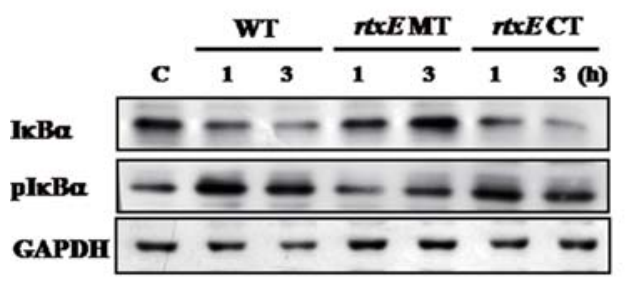

B

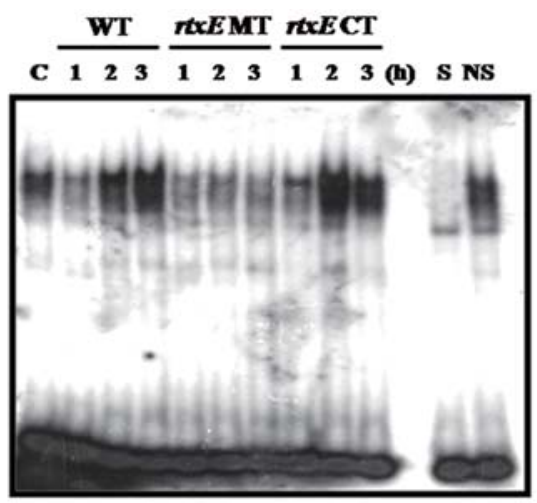

D

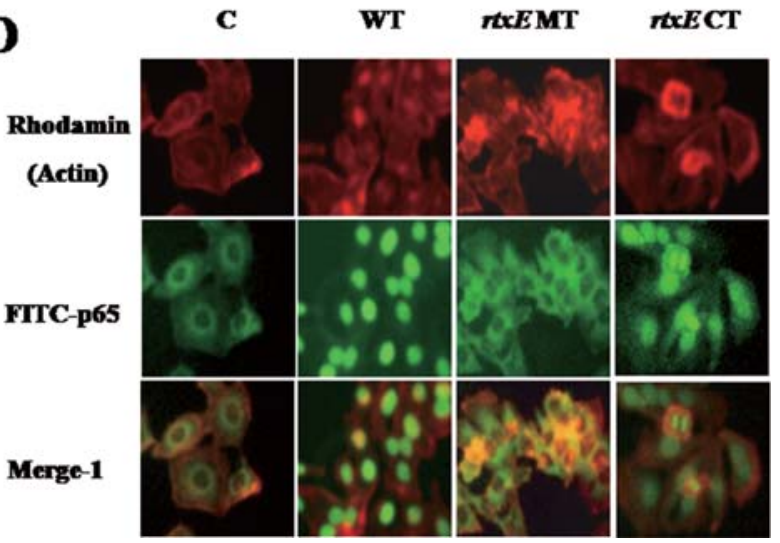

Figure 6. Effects of co-culture supernatants from $r t x E$ CT $V$. vulnificus-infected INT-407 cells on NF-кB DNA activation and IкB $\alpha$ phosphorylation. (A) Human intestinal epithelial cells were transiently co-transfected with the NF-кB minimal promoter/luciferase construct and pRL-TK reporter vector, followed by 12 or $24 \mathrm{~h}$ of treatment with co-culture supernatants from WT, rtxE MT, or rtxE CT V. vulnificus-infected INT-407 cells. Afterwards, the cells were washed with PBS and the luciferase activity was determined. The results were normalized by Renilla luciferase activity, and are expressed as the relative fold induction. The data are representative of 3 independent experiments. The data are expressed as the means \pm standard errors $\left(\mathrm{n}=3\right.$ ). ${ }^{*} \mathrm{P}<0.01$, relative to the group treated with co-culture supernatants from WT $V$. vulnificus-infected INT-407 cells at $24 \mathrm{~h} .{ }^{* *} \mathrm{P}<0.05$, relative to the group treated with co-culture supernatants from rtxE MT V. vulnificus-infected INT-407 cells at $24 \mathrm{~h}$. (B) Human intestinal epithelial cells were treated for 1-3 h with co-culture supernatants from WT, rtxE MT, or rtxE CT V. vulnificus-infected INT-407 cells. NF-кB DNA binding activity in the nuclear extracts was assessed via EMSA. S (specific) and NS (non-specific) indicate the presence of a 50-fold excess of specific oligonucleotide (NF- $\mathrm{B}$ ) and non-specific oligonucleotide (CRE-containing oligonucleotide), respectively. (C) Human intestinal epithelial cells were treated with co-culture supernatants from WT, rtxE MT, or $r t x E$ CT V. vulnificus-infected INT-407 cells. The cell lysates were prepared and analyzed by Western blot analysis using anti-IкB $\alpha$, anti-pIкB $\alpha$ and $B$-actin antibodies. The data are representative of 3 independent experiments. (D) Reduced NF-кB p65 nuclear translocation in human intestinal epithelial cells treated with coculture supernatants from rtxE MT V. vulnificus-infected INT-407 cells. Human intestinal epithelial cells grown in 24 -well plates were treated for 3 h with coculture supernatants from WT, rtxE MT, or rtxE CT V. vulnificus-infected INT-407 cells. The cells were washed, fixed, permeabilized, and stained with antip65 polyclonal antibody followed by staining with FITC-conjugated secondary antibody (green) or rhodamine phalloidin (red; actin staining). NF- $\mathrm{B}$ p65-stained cells were merged with rhodamine phalloidin actin staining. The data are representative of 3 independent experiments. WT, wild-type; $r t x E$ MT, $r t x E$ mutant; rtxE CT, rtxE-complemented.

The reduced levels of $I \kappa B \alpha$ phosphorylation and $N F-\kappa B$ translocation were restored by co-culture supernatants from rtxE-complemented $V$. vulnificus-infected INT-407 cells. In order to further verify whether the reduced NF- $\mathrm{KB}$ activity was restored by co-culture supernatants from the rtxEcomplemented $V$. vulnificus-infected INT-407 cells, the effects of co-culture supernatants from the rtxE-complemented

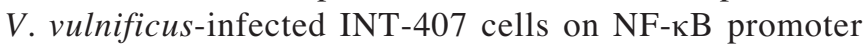

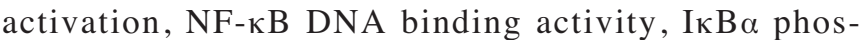
phorylation and the nuclear translocation of $\mathrm{NF}-\kappa \mathrm{B}$, were evaluated. Human intestinal epithelial cells were incubated for $3 \mathrm{~h}$ with co-culture supernatants from wild-type, $r t x E$ mutant, or rtxE gene-complemented $V$. vulnificus-infected INT-407 cells. As shown in Fig. 6, co-culture supernatants from the rtxE mutant $V$. vulnificus-infected INT-407 cells induced significantly lower levels of NF- $\mathrm{NB}$ promoter

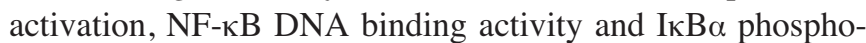
rylation compared to those from the wild-type $V$. vulnificusinfected INT-407 cells. The reduced levels were restored when the cells were treated with co-culture supernatants from the rtxE-complemented $V$. vulnificus-infected INT-407 cells. Furthermore, as shown in Fig. 6D, the immunofluorescence staining revealed the translocation of $\mathrm{NF}-\mathrm{\kappa B}$ into the nuclei of human intestinal epithelial cells after treatment with coculture supernatants from either the wild-type or $r t x E$ complemented $V$. vulnificus-infected INT-407 cells. In contrast, NF- $\mathrm{\kappa B}$ was predominantly localized in the cytoplasm when intestinal epithelial cells were treated with coculture supernatants from rtxE mutant $V$. vulnificus-infected INT-407 cells. Additionally, as shown in Fig. 7, treatment 


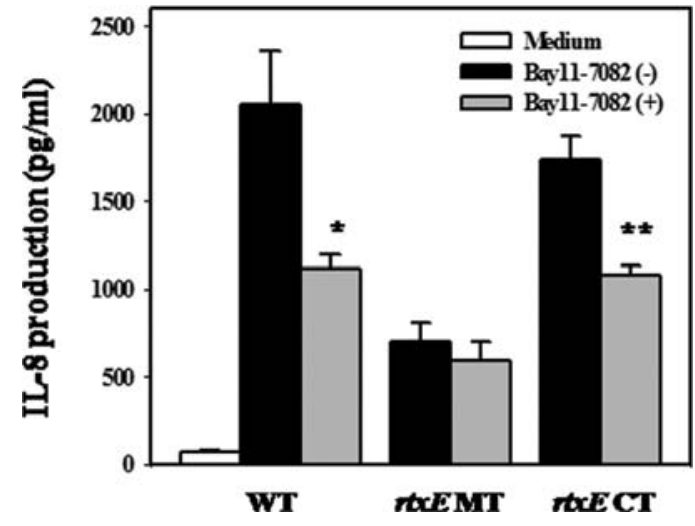

Figure 7. Effects of the NF-кB inhibitors on IL-8 production induced by coculture supernatants from $V$. vulnificus-infected host cells. Human intestinal epithelial cells were pre-treated for $1 \mathrm{~h}$ with the NF- $\mathrm{BB}$ inhibitor BAY11-7082 $(20 \mu \mathrm{M})$, followed by treatment with co-culture supernatants $(1.5 \mathrm{ml} /$ well, 6-well plate) from $V$. vulnificus-infected INT-407 cells for $24 \mathrm{~h}$. The supernatants were analyzed for the IL- 8 protein by ELISA. The data are representative of 3 independent experiments. The data represent the mean \pm standard errors $(n=3) .{ }^{*} \mathrm{P}<0.01$, relative to the group treated with co-culture supernatants from WT $V$. vulnificus-infected INT-407 cells without BAY117082. ${ }^{* *} \mathrm{P}<0.01$, relative to the group treated with co-culture supernatants from rtxE CT $V$. vulnificus-infected INT-407 cells without BAY11-7082. WT, wild-type; rtxE CT, rtxE-complemented.

with BAY11-7082, a specific inhibitor of NF- $\mathrm{KB}$ activation, resulted in a significant diminution of IL-8 production induced by co-culture supernatants from the wild-type or $r x E$ complemented V. vulnificus-infected INT-407 cells (Fig. 7). The results clearly indicate that co-culture supernatants from $V$. vulnificus-infected INT-407 cells are involved in the production of IL-8 in human intestinal epithelial cells via $\mathrm{NF}-\kappa \mathrm{B}$ activation when the bacteria come into contact with the host cells.

\section{Discussion}

In this study, we hypothesized that the TISS transporter system of $V$. vulnificus could actively secrete factors into the supernatant when the bacteria make contact with host cells. Also, $V$. vulnificus causes the secretion of factors from host cells to the culture medium. The secreted factors from the bacteria and host cells can induce IL-8 production in human intestinal epithelial cells. Our results clearly demonstrate that co-culture supernatants from the rtxE mutant of $V$. vulnificus significantly induce lower levels of IL- 8 production and NF- $\mathrm{KB}$ activity compared to those from the wild-type $V$. vulnificus-infected INT-407 cells, and that the reduction in levels induced by coculture supernatants from the rtxE mutant $V$. vulnificusinfected INT-407 cells was restored by those from the $r t x E$ complemented V. vulnificus-infected INT-407 cells, thereby indicating that the rtxE mutant of $V$. vulnificus was defective in secreting factors involved in IL-8 production, whereas the wild-type of $V$. vulnificus could secrete those factors when co-cultured with INT-407 cells.

It remains unclear as to which factors are secreted into the supernatant via the TISS of $V$. vulnificus when the bacteria make direct contact with the host cells. Proteomic analysis studies are currently underway to compare the co-culture supernatants from wild-type, $r t x E$ mutant, or $r t x E$-complemented $V$. vulnificus-infected INT-407 cells. The RtxA toxin is known to be secreted via the TISS, and its secretion has been shown to require the direct exposure of $V$. vulnificus to the host cells (11). The RTX toxin induces cell rounding and increased permeability through tight cellular junctions due to the cross-linking of actin monomers, thus resulting in the depolymerization of actin stress fibers (25). In a murine pulmonary cholera model, the RTX toxin mutants have demonstrated less severe pathology and reduced serum levels of pro-inflammatory IL-6 and murine macrophage inflammatory protein-2, thereby suggesting that RTX is relevant to the severity of acute inflammatory responses (26). One consequence of cytolysis or cell damage is the release of intracellular contents, including cytoplasmic proteins, into the extracellular space and peripheral circulation (27-29). An accumulation of secreted extracellular proteins from the host can contribute to increased blood viscosity, endothelial damage and microvascular thrombosis (30). Therefore, we assume that the expressions of pro-inflammatory cytokines are induced by the virulence factors of $V$. vulnificus, including RTX toxins, and secreted endogenous proteins from the host.

The mechanism by which co-culture supernatants from rtxE mutant $V$. vulnificus-infected INT-407 cells, compared to those from wild-type $V$. vulnificus-infected INT-407 cells induced significant reductions in IL-8 production at the molecular level, remains to be clearly elucidated. However, several lines of evidence support the notion that the reduced IL-8 production described herein is closely correlated with the down-regulation of $\mathrm{NF}-\kappa \mathrm{B}$ activity in human intestinal epithelial cells treated with co-culture supernatants from $\mathrm{rtxE}$ mutant V. vulnificus-infected INT-407 cells. Co-culture supernatants from the rtxE mutant $V$. vulnificus-infected INT-407 cells induced significantly lower levels of NF-кB

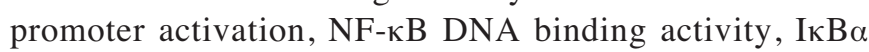
phosphorylation and NF- $\kappa \mathrm{B}$ nuclear translocation than those from the wild-type $V$. vulnificus-infected INT-407 cells. Additionally, co-culture supernatants from the wild-type $V$. vulnificus-infected INT-407 cells strongly stimulated IL-8 promoter activation, and its stimulatory effect disappeared in the cells transfected with the IL-8/LS promoter harboring the LS mutation of the NF-кB sites. Finally, the reduced levels of NF- $\mathrm{BB}$ activities attributable to the co-culture supernatants from the rtxE mutant $V$. vulnificus-infected INT-407 cells were restored by those from the rtxE-complemented $V$. vulnificusinfected INT-407 cells. These results clearly indicate that the stimulatory effects of co-culture supernatants from the $V$. vulnificus-infected INT-407 cells on IL-8 production, were mediated via the $\kappa \mathrm{B}$ site. We have previously reported that infection with $V$. vulnificus induced IL-8 production in human intestinal epithelial cells via NF- $\mathrm{BB}$ activation $(6,17)$.

The results of the present study clearly demonstrate for the first time that RtxE, a transporter of cytotoxic factors, performs a crucial role in secreting factors from bacteria and host cells involved in the IL-8 production of human intestinal epithelial cells exposed to $V$. vulnificus. The results suggest that RtxE could possibly be utilized as a target in the regulation of $V$. vulnificus pathogenesis. 


\section{Acknowledgements}

This study was supported by grants from the Korea Research Foundation funded by the Korean Government (no. KRF2008-314-C00336), and the Marine Biotechnology Program funded by the Ministry of Land, Transport and Maritime Affairs (no. 0552-200700001).

\section{References}

1. Jeong HS, Lee MH, Lee KH, Park SJ and Choi SH: SmcR and cyclic AMP receptor protein coactivate Vibrio vulnificus $\mathrm{vvpE}$ encoding elastase through the RpoS-dependent promoter in a synergistic manner. J Biol Chem 278: 45072-45081, 2003.

2. Kumamoto KS and Vukich DJ: Clinical infections of Vibrio vulnificus: a case report and review of the literature. J Emerg Med 16: 61-66, 1993.

3. Strom MS and Paranjpye RN: Epidemiology and pathogenesis of Vibrio vulnificus. Microbes Infect 2: 177-188, 2000

4. Powell JL, Wright AC, Wasserman SS, Hone DM and Morris JG Jr: Release of tumor necrosis factor alpha in response to Vibrio vulnificus capsular polysaccharide in vivo and in vitro models. Infect Immun 65: 3713-3718, 1997.

5. Wright AC, Powell JL, Kaper JB and Morris JG Jr: Identification of a group 1-like capsular polysaccharide operon for Vibrio vulnificus. Infect Immun 69: 6893-6901, 2001.

6. Lee BC, Kim MS, Choi SH and Kim TS: Involvement of capsular polysaccharide via a TLR2/NF- $\mathrm{kB}$ pathway in Vibrio vulnificus-induced IL-8 secretion of human intestinal epithelial cells. Int J Mol Med 25: 581-591, 2010.

7. Gray LD and Kreger AS: Purification and characterization of extracellular cytolysin produced by Vibrio vulnificus. Infect Immun 48: 67-72, 1985

8. Simpson LM and Oliver JD: Siderophore production by Vibrio vulnificus. Infect Immun 41: 644-649, 1983.

9. Paul AG, Keri LB and Angela MS: Molecular pathogenesis of Vibrio vulnificus. J Microbiol 43: 118-131, 2005.

10. Lee JH, Kim MW, Kim BS, Kim SM, Lee BC, Kim TS and Choi SH: Identification and characterization of the Vibrio vulnificus rtxA essential for cytotoxicity in vitro and virulence in mice. J Microbiol 45: 146-152, 2007.

11. Lee BC, Lee JH, Kim MW, Kim BS, Oh MH, Kim KS, Kim TS and Choi SH: Vibrio vulnificus rtxE is important for virulence, and its expression is induced by exposure to host cells. Infect Immun 76: 1509-1517, 2008.

12. Lee BC, Choi SH and Kim TS: Vibrio vulnificus RTX toxin plays an important role in the apoptotic death of human intestinal epithelial cells exposed to Vibrio vulnificus. Microbes Infect 10: 1504-1513, 2008.

13. Boardman BK and Satchell KJ: Vibrio cholerae strains with mutations in an atypical type I secretion system accumulate RTX toxin intracellularly. J Bacteriol 186: 8137-8143, 2004.

14. Sheahan KL, Cordero CL and Satchell KJ: Identification of a domain within the multifunctional Vibrio cholerae RTX toxin that covalently cross-links actin. Proc Natl Acad Sci USA 101: 9798-9803, 2004.

15. Fuller KJ and Mekalanos JJ: In vivo covalent cross-linking of cellular actin by the Vibrio cholerae RTX toxin. EMBO J 12: 5315-5323, 2000.
16. Espat NJ, Auffenberg T, Abouhamze A, Baumhofer J, Moldawer LL and Howard RJ: A role for tumor necrosis factor- $\alpha$ in the increased mortality associated with Vibrio vulnificus infection in the presence of hepatic dysfunction. Ann Surg 223: 428-433, 1996.

17. Lee BC, Kim SH, Choi SH and Kim TS: Induction of interleukin-8 production via nuclear factor $-\mathrm{\kappa}$ activation in human intestinal epithelial cells infected with Vibrio vulnificus. Immunology 115: 506-515, 2005.

18. Morrison DC and Ryan JL: Endotoxins and disease mechanisms. Annu Rev Med 38: 417-432, 1987.

19. Hesse DG, Tracey KJ, Fon Y, Manogue KR, Paladino MA Jr, Cerami A, Shires GT and Lowry SF: Cytokine appearance in human endotoxemia and primate bacteremia. Surg Gynecol Obstet 166: 147-153, 1998.

20. Morrison DC, Danner RL, Dinarello CA, Munford RS, Natanson C, Pollack M, Spitzer JA, Ulevitch RJ, Vogel SN and McSweegan E: Bacterial endotoxins and pathogenesis of Gramnegative infections: current status and future directions. J Endotoxin Res 1: 71-83, 1994.

21. Beutler B, Milsark IW and Cerami A: Passive immunization against cachectin/tumor necrosis factor protects mice from lethal effects of endotoxin. Science 229: 869-871, 1985.

22. Gerard C, Bruyns C, Marchant A, Abranmowitz D, Vandenbabeele P, Delvaux A, Fiers W, Goldman M and Velu T: Interleukin 10 reduces the release of tumor necrosis factor and prevents lethality in experimental endotoxemia. J Exp Med 177: 1205-1208, 1993.

23. Jung YD, Fan F, McConkey DJ, Jean ME, Liu W, Reinmuth N, Stoeltzing O, Ahmad SA, Parikh AA, Mukaida N and Ellis LM: Role of p38 MAPK, AP-1, and NF-кB in interleukin-1-induced IL-8 expression in human vascular smooth muscle cells. Cytokine 18: 206-213, 2002.

24. Matsusaka T, Fujikawa K, Nishio Y, Mukaida N, Matsushima K, Kishimoto T and Akira ST: Transcription factors NF-IL6 and $\mathrm{NF}-\mathrm{kB}$ synergistically activate transcription of the inflammatory cytokines, interleukin-6 and interleukin-8. Proc Natl Acad Sci USA 90: 10193-10197, 1993.

25. Lin W, Fullner KJ, Clayton R, Sexton JA, Rogers MB, Calia KE, Calderwood SB, Fraser C and Mekalanos JJ: Identifiaction of a Vibrio cholerae RTX toxin gene cluster that is tightly linked to the cholera toxin prophage. Proc Natl Acad Sci USA 96: 1071-1076, 1999.

26. Fullner KM, Boucher JC, Hanes MA, Haines GK, Meehan BM, Walchle C, Sansonetti PJ and Mekalanos JJ: The contribution of accessory toxins of Vibrio cholerae O1 E1 Tor to the proinflammatory response in a murine pulmonary cholera model. J Exp Med 195: 1455-1462, 2002.

27. Clinkenbeard KD, Mosier DA, Timko AL and Confer AW: Effects of Pasteurella haemolytica leukotoxin on cultured bovine lymphoma cells. Am J Vet Res 50: 271-275, 1989.

28. Smith DB, Janmey PA and Lind SE: Circulating actin-gelsolin complexes following oleic acid-induced lung injury. Am J Pathol 130: 261-267, 1988.

29. Goldschmidt-Clermont PJ, Lee WM and Galbraith RM: Proportion of circulating Gc (vitamin D-binding protein) in complexed form: relation to clinical outcome in fulminant hepatic failure. Gastroenterology 94: 1454-1458, 1988.

30. Haddad JG, Harper KD, Guoth M, Pietra GG and Sanger JW: Angiopathic consequences of saturating the plasma scavenger system for actin. Proc Natl Acad Sci USA 87: 1381-1385, 1990. 